\title{
Características de la saliva en niños con Síndrome de Down
} Pinzón-Te $\mathrm{AL}^{1}$, López-Pérez $\mathrm{R}^{2}$, Enríquez-Rivera $\mathrm{FM}^{3}$, Aguilar-Ayala FJ ${ }^{4}$, Rejón-
Peraza $\mathrm{ME}^{5}$, López-Osorio $\mathrm{JL}^{6}$

\section{Resumen}

ANTECEDENTES: los niños con Síndrome de Down (SD) generalmente tienen características morfogenéticas que los predisponen a patologías bucales; por lo tanto, el análisis de las características de la saliva constituye una herramienta diagnóstica de su estado de salud bucal.

OBJETIVOS: a) Comparar los valores promedio de $\mathrm{pH}$ salival y tasa de flujo salival (TFS), según género y edad. b) Comparar los tipos de $\mathrm{pH}$ salival y TFS, según género y edad. c) Determinar si existe correlación entre el pH salival y la TFS.

MATERIALES Y MÉTODOS: en 100 pacientes con SD $(M=50, F=50)$ de 0 a 18 años de edad pertenecientes a instituciones de educación especial de Yucatán. Se midió el pH salival mediante un pHmetro digital portátil ATS $^{\circledR}$ y se determinó la TFS mediante la técnica de pesada de algodón Strongin, Hinsie y Peck.

RESULTADOS: se encontraron diferencias estadísticamente significativas entre los tipos de $\mathrm{pH}$ por edad $\left(\mathrm{X}^{2}=27.333 ; \mathrm{p}<0.001\right)$ y en los valores promedio de la TFS por género $(t=2.125 ; \mathrm{p}=0.036)$.

CONCLUSIÓN: los valores de pH salival y TFS deben ser considerados como indicadores de salud durante la consulta odontológica del paciente con SD.

PALABRAS CLAVE: pH salival, flujo salival, síndrome de Down.

\section{Salivary characteristics in Down's Syndrome children.}

Pinzón-Te $A L^{1}$, López-Pérez $\mathrm{R}^{2}$, Enríquez-Rivera $\mathrm{FM}^{3}$, Aguilar-Ayala $\mathrm{FJ}^{4}$, RejónPeraza $\mathrm{ME}^{5}$, López-Osorio $\mathrm{JL}^{6}$

\footnotetext{
Abstract

BACKGROUND: Children with Down`s Syndrome (DS) have morphogenetic characteristics predisposing to oral diseases. It is also important to consider that the analysis of the characteristics of saliva are a diagnostic tool for appraisal oral health.
}

${ }^{1}$ Departamento de Odontología Infantil, Facultad de Odontología, Universidad Autónoma de Yucatán, México.

${ }^{2}$ Departamento de Salud Pública Bucal, Facultad de Odontología, Universidad Nacional Autónoma de México, México.

${ }^{3}$ Ex residente de la Maestría en Odontología Infantil, Facultad de Odontología, Universidad Autónoma de Yucatán, México.

${ }^{4}$ Departamento de Odontología Infantil, Facultad de Odontología, Universidad Autónoma de Yucatán, México.

${ }^{5}$ Departamento de Odontología Infantil, Facultad de Odontología, Universidad Autónoma de Yucatán, México.

${ }^{6}$ Especialista en Estadística, Universidad Latino, México.

Recibido: 24 de agosto del 2016

Aceptado: 10 de febrero del 2017

\section{Correspondencia}

Alicia Leonor Pinzón Te

alicia.pinzon@correo.uady.mx

\section{Este artículo debe citarse como}

Pinzón-Te AL, López-Pérez R, Enríquez-Rivera FM, Aguilar-Ayala FJ, Rejón-Peraza ME, López-Osorio JL. Características de la saliva en niños con Síndrome de Down. Acta Pediatr Mex. 2017;38(6):355-362. 
OBJECTIVES: a) To compare the average values of salivary $\mathrm{pH}$ and salivary flow rate (SFR) by gender and age. b) To compare the type of salivary $\mathrm{pH}$ and SFR by gender and age. c) To determine whether there was a correlation between the salivary $\mathrm{pH}$ and the SFR.

MATERIAL AND METHODS: In 100 patients with DS ( $M=50, F=50)$ from 0 to 18 years of age, belonging to special education institutions of Yucatan, salivary $\mathrm{pH}$ was measured by a portable digital $\mathrm{pH}$ meter $\mathrm{ATS}^{\circledR}$; SFR was obtained by the technique of heavy cotton Strongin, Hinsie and Peck.

RESULTS: Statistically significant differences were found among the types of $\mathrm{pH}$ according to age $\left(\mathrm{X}^{2}=27,333 ; \mathrm{p}<0.001\right)$, as well as among the average values of the SHP according to gender $(t=2.125$, $p=0.036)$.

CONCLUSION: The values of the salivary $\mathrm{pH}$ and salivary flow should be considered as oral health indicators in patients with DS.

KEYWORDS: salivary pH; salivary flow; Down's syndrome
Correspondence Alicia Leonor Pinzón Te alicia.pinzon@correo.uady.mx

\section{INTRODUCCIÓN}

El Síndrome de Down (SD) se considera dentro de las causas más frecuentes de discapacidad intelectual en México. De acuerdo con el Instituto Nacional de Estadística y Geografía (INEGI), la incidencia en Yucatán es de 1/550 recién nacidos vivos, lo que lo convierte en el estado con mayor prevalencia de discapacidad intelectual en México. Los pacientes con SD también tienen alteraciones morfológicas y fisiológicas en diversas partes de su organismo y el sistema estomatognático no es la excepción. Estas alteraciones constituyen factores de riesgo para patologías bucales tales como la caries dental y la enfermedad periodontal. ${ }^{1}$

Entre las alteraciones del sistema estomatognático se encuentran las alteraciones morfogenéticas de los maxilares y la lengua, la hipotonía muscular, así como por la ingesta de medicamentos y de alimentos blandos de consistencia pegajosa. Todas ellas pueden favorecer las condiciones locales adversas tales como el estancamiento de alimentos y la distribución inadecuada de la saliva con la consecuente disminución en su acción lítica en la cavidad bucal. De igual manera, pueden provocar un valor del $\mathrm{pH}$ bucal demasiado ácido, lo cual exacerba la caries dental o por el contrario, muy alcalino, lo que favorece el desarrollo de la enfermedad periodontal. ${ }^{2}$

La tasa de flujo salival (TFS), es decir, la cantidad de saliva en mililitros $(\mathrm{mL})$ que el organismo produce por minuto $(\mathrm{min})$, modifica las propiedades físicas y químicas de la saliva y favorece sus propiedades líticas, lo que mantiene la concentración de iones de hidrógeno o potencial de hidrógeno $(\mathrm{pH})$ lo más cercano a la neutralidad y logra así un estado óptimo de calidad. Lo anterior promueve a su vez un adecuado ejercicio de sus funciones protectoras frente a agresores, desde su 
función en la formación del bolo alimenticio hasta su acción neutralizadora de ácidos. La saliva es entonces, un factor protector y de mantenimiento de la homeostasis en la cavidad bucal; a medida que se secreta, se mezcla con otros elementos locales de la cavidad bucal y genera condiciones adecuadas para la vida microbiana. Respecto del $\mathrm{pH}$ salival y TFS, se ha descrito en la literatura una diferencia mínima entre los valores de $\mathrm{pH}$ salival de niños y niñas con SD y la TFS disminuye a mayor edad en estos niños. ${ }^{3-5}$

\section{OBJETIVOS}

a) Comparar los valores promedio del $\mathrm{pH}$ salival y TFS según género y edad. b) Comparar los tipos de $\mathrm{pH}$ salival y TFS según género y edad, c) Determinar si existió correlación entre el pH salival y la TFS.

\section{MATERIALES Y MÉTODOS}

Se realizó un estudio de tipo transversal, observacional con apego a los principios éticos enunciados en la Declaración de Helsinki. La muestra estuvo conformada por 100 niños con $\mathrm{SD}(\mathrm{M}=50, \mathrm{~F}=50)$ entre 0 y 18 años de edad, inscritos en seis escuelas de educación especial, ubicados en el municipio de Mérida, Yucatán, México, que cumplieron con los criterios de inclusión y de exclusión. Se consideraron como criterios de inclusión que los niños no presentaran enfermedades sistémicas secundarias, que no hubieran ingerido medicamentos dos semanas previas a la toma de la muestra de saliva y que los padres hubieran firmado la carta de consentimiento informado. Como criterios de exclusión, que los niños se encontraran en tratamiento de ortodoncia o que no cooperaran durante la toma de la muestra de saliva. ${ }^{6,7}$

Para el análisis por edad, se formaron arbitrariamente cinco grupos de edad: 0-3 años; 4-7 años; 8-11 años; 12-15 años y 16-18 años.
La recolección de los datos se realizó de diciembre de 2013 a agosto 2014, en un horario de 15:00 a 19:00 hrs. Todos los niños al momento de la realización de los procedimientos de recolección tenían por lo menos dos horas de ayuno, desde su último cepillado dental.

Un solo examinador efectuó todas las mediciones, previamente estandarizado y con un nivel de confiabilidad intraexaminador de 1.0 para la medición del pH salival y de 0.97 para la medición de la TFS, de acuerdo con el coeficiente Kappa de Cohen. Se asignó un folio a cada niño en el que se registró su nombre, edad y género. Se colocó a los niños en una silla recta, a continuación se colectó saliva mixta no estimulada mediante la técnica simplificada de Strongin, Hinsie y Peck, la cual consistió en pesar previamente tres rollos de algodón de tamaño estándar de casi $4 \mathrm{~cm}$ (1.5 pulgadas) de largo tomados de un recipiente hermético; luego se colocó uno de ellos en la zona sublingual y dos en la zona vestibular en ambos lados durante dos minutos. Posteriormente, se pesaron nuevamente los rollos de algodón y la cantidad de flujo salival se consideró como la semidiferencia entre el peso de antes y después de la recolección salival (tiempo en minutos de colección salival) expresados en $\mathrm{mL} / \mathrm{min}^{8,9}$

$$
T F S=\frac{P F A-P I A}{2 \text { minutos }}=(X) \mathrm{mL} / \mathrm{min}
$$

Donde: PFA = peso final del algodón $(\mathrm{gr}=\mathrm{mL})$; $\mathrm{PIA}=$ peso inicial del algodón $(\mathrm{gr}=\mathrm{mL})$.

Los resultados se expresaron en $\mathrm{mL} / \mathrm{min}$ de saliva, pues volumétricamente es igual a gr/min dado que la saliva tiene una composición de 99\% o más de agua, y se clasificaron como: 1 ) Xerostomía, los valores de $0.1 \mathrm{~mL} / \mathrm{min}$ o menos. 2) Flujo pronunciadamente bajo, valores 0.101 $-0.7 \mathrm{~mL} / \mathrm{min}$. 3) Flujo bajo, de $0.701-1.0 \mathrm{~mL} / \mathrm{min}$. 4) Flujo normal, $1.01-2.0 \mathrm{~mL} / \mathrm{min}$. 5) Hipersalivación, de $2.0 \mathrm{~mL} / \mathrm{min}$ o más. 
Posteriormente, se utilizó un pHmetro digital para líquidos marca $\mathrm{ATS}^{\circledR}$, previamente calibrado. La parte activa del pHmetro se ubicó en contacto íntimo con la zona ventral de la lengua durante 5 segundos, y se tomó el valor mínimo y constante durante 5 segundos más. Se registraron los resultados en unidades y décimas de unidades y se clasificaron los valores como: alcalino $>7.1$, neutro $=7$ y ácido $<6.9$.

Los datos obtenidos se analizaron con el programa estadístico SPSS versión 22.0, y se aplicaron las siguientes pruebas estadísticas: ji cuadrada $\left(\mathrm{X}^{2}\right)$ para determinar si existió una diferencia estadísticamente significativa entre los porcentajes de los diferentes tipos de $\mathrm{pH}$ y TFS por género y edad; $t$ de Student (t) para la misma determinación al comparar los niveles promedio de $\mathrm{pH}$ y TFS por género; ANOVA de una vía (F) para realizar lo propio con los niveles promedio de $\mathrm{pH}$ y TFS entre los diferentes grupos etáreos y; finalmente, se aplicó el coeficiente de correlación de Pearson (r) para determinar si se presentó una correlación entre el nivel de $\mathrm{pH}$ y la TFS.

\section{RESULTADOS}

pH salival

El valor promedio de $\mathrm{pH}$ salival en la muestra fue de 6.89 con una DE de 0.558; el valor mínimo encontrado fue 5.2 y el máximo de 8.6.

El valor mínimo de $\mathrm{pH}$ salival en el género masculino fue de 5.4 y el máximo fue de 8.6. En el género femenino los valores fueron de 5.2 y 7.9 , respectivamente.

No obstante, al aplicar la prueba t de Student para comparar los valores de $\mathrm{pH}$ por género, se observó que si bien este promedio fue ligeramente mayor en el género femenino, no hubo una diferencia estadísticamente significativa entre ambos géneros (Cuadro 1).
Cuadro 1. Promedio del $\mathrm{pH}$ salival por género

\begin{tabular}{|l|c|c|c|c|c|} 
& \multicolumn{2}{|c|}{ pH salival } & \multicolumn{3}{|c}{} \\
\hline Género & Media & DE & T & IC 95\% & Sig. \\
\hline $\begin{array}{l}\text { Masculino } \\
(\mathrm{n}=50)\end{array}$ & 6.878 & 0.524 & & & \\
$\begin{array}{l}\text { Femenino } \\
(\mathrm{n}=50)\end{array}$ & 6.912 & 0.596 & 0.143 & $\begin{array}{c}-0.237- \\
0.205\end{array}$ & 0.887 \\
$\begin{array}{l}\text { Total } \\
(\mathrm{N}=100)\end{array}$ & 6.895 & 0.558 & & & \\
\end{tabular}

Al comparar los valores del $\mathrm{pH}$ de los cinco grupos de edad con la prueba de ANOVA, no se encontró una diferencia estadísticamente significativa entre ellos, a pesar de que el valor promedio del $\mathrm{pH}$ salival tendió a elevarse ligeramente conforme aumentaba la edad (Cuadro 2).

Cuadro 2. Promedio del pH salival en ambos géneros de acuerdo con la edad

\begin{tabular}{|l|c|c|c|c|c|}
\hline \multicolumn{2}{|c|}{} & \multicolumn{2}{c|}{ pH salival } & \multicolumn{2}{c}{} \\
\hline Edad (años) & $\mathbf{n}$ & Media & DE & F & Sig. \\
\hline $0-3$ & 5 & 6.76 & 0.23 & & \\
\hline $4-7$ & 22 & 6.786 & 0.506 & & \\
\hline $8-11$ & 38 & 6.811 & 0.614 & 1.611 & 0.178 \\
\hline $12-15$ & 24 & 7.021 & 0.606 & & \\
$16-18$ & 11 & 7.191 & 0.308 & & \\
Total & 100 & 6.895 & 0.558 & & \\
\hline
\end{tabular}

Al clasificar los valores del pH salival en ácido, neutro y alcalino se observó que en ambos géneros predominó un $\mathrm{pH}$ ácido; sin embargo, no hubo una diferencia estadísticamente significativa entre los porcentajes de ambos géneros en relación al tipo de $\mathrm{pH}$ salival al aplicar la prueba $\mathrm{X}^{2}$ (Cuadro 3).

Asimismo, cuando se analizó el tipo de pH salival como ácido, neutro o alcalino entre los 
Cuadro 3. Frecuencias de los tipos del pH salival de acuerdo con el género

\begin{tabular}{|c|c|c|c|c|c|c|}
\hline & & \multicolumn{3}{|c|}{ Tipo de pH } & \multirow[b]{2}{*}{$\mathrm{X}^{2}$} & \multirow[b]{2}{*}{ Sig. } \\
\hline Género & $\mathbf{N}$ & Ácido & Neutro & Alcalino & & \\
\hline Masculino & 50 & 30 & 4 & 16 & \multirow{3}{*}{2.905} & \multirow{3}{*}{0.234} \\
\hline Femenino & 50 & 27 & 1 & 22 & & \\
\hline Total & 100 & 57 & 5 & 38 & & \\
\hline
\end{tabular}

diferentes grupos de edad, se observó que de los 0 a los 11 años predominó el tipo ácido; mientras que de los 16 a los 18 años el mayor porcentaje correspondió al tipo alcalino, observándose una diferencia estadísticamente significativa entre los porcentajes de acuerdo al grupo de edad al aplicar la prueba de $X^{2}$ (Cuadro 4).

Cuadro 4. Frecuencias de los tipos del pH salival de acuerdo con la edad

\begin{tabular}{|c|c|c|c|c|c|c|}
\hline \multirow[b]{2}{*}{$\begin{array}{l}\text { Edad } \\
\text { (años) }\end{array}$} & \multicolumn{4}{|c|}{ Tipo de pH salival } & \multirow[b]{2}{*}{$X^{2}$} & \multirow[b]{2}{*}{ Sig. } \\
\hline & $\mathbf{n}$ & Ácido & Neutro & $\begin{array}{l}\text { Alca- } \\
\text { lino }\end{array}$ & & \\
\hline $0-3$ & 5 & 4 & 1 & 0 & \multirow{6}{*}{27.333} & \multirow{6}{*}{$<0.001^{*}$} \\
\hline $4-7$ & 22 & 15 & 0 & 7 & & \\
\hline $8-11$ & 38 & 25 & 1 & 12 & & \\
\hline $12-15$ & 24 & 12 & 0 & 12 & & \\
\hline $16-18$ & 11 & 1 & 3 & 7 & & \\
\hline Total & 100 & 57 & 5 & 38 & & \\
\hline
\end{tabular}

*Diferencia estadísticamente significativa.

\section{TFS}

El valor mínimo de la TFS en la muestra se ubicó en $0.050 \mathrm{~mL} / \mathrm{min}$ y el máximo a $1.150 \mathrm{~mL} / \mathrm{min}$. El promedio total de la muestra para esta variable fue de $0.410 \mathrm{~mL} / \mathrm{min}$ con una DE de 0.230 . El género masculino tuvo un valor mínimo de $0.050 \mathrm{~mL} / \mathrm{min}$ y un máximo de $1.150 \mathrm{~mL} / \mathrm{min}$, mientras que en el género femenino fueron $0.070 \mathrm{~mL} / \mathrm{min}$ y $0.990 \mathrm{~mL} / \mathrm{min}$, respectivamente.
Al realizar la estratificación por género para el análisis de esta variable, el mayor promedio se presentó en el género masculino con una diferencia estadísticamente significativa en relación al género femenino, como lo indica la prueba $t$ de Student (Cuadro 5).

Cuadro 5. Promedio de la TFS por género

\begin{tabular}{|l|c|c|c|c|c|}
\hline & \multicolumn{2}{|l|}{ TFS (mL/min) } & & & \\
\hline Género & Media & DE & $\mathbf{t}$ & IC 95\% & Sig. \\
\hline $\begin{array}{l}\text { Masculino } \\
(\mathrm{n}=50)\end{array}$ & 0.458 & 0.249 & & & \\
$\begin{array}{l}\text { Femenino } \\
(\mathrm{n}=50)\end{array}$ & 0.362 & 0.2 & 2.125 & $0.006-0.185$ & $0.036^{*}$ \\
$\begin{array}{l}\text { Total } \\
(\mathrm{N}=100)\end{array}$ & 0.41 & 0.23 & & & \\
\hline
\end{tabular}

*Diferencia estadísticamente significativa.

En cambio, al analizar esta variable de acuerdo a la edad, aplicando la prueba ANOVA, no se encontraron diferencias significativas entre el promedio obtenido en cada uno de los grupos de edad (Cuadro 6).

En este estudio se consideraron cinco tipos de flujo salival: xerostomía, pronunciadamente bajo, bajo, normal e hipersalivación. En el total de la muestra estudiada se reveló un predominio absoluto del tipo pronunciadamente bajo; en cambio, no hubo ningún caso de hipersalivación.

Cuadro 6. Promedio de la TFS en ambos géneros de acuerdo con la edad

\begin{tabular}{|l|c|c|c|c|c|}
\hline & & \multicolumn{2}{|c|}{ TFS $(\mathrm{mL} / \mathrm{min})$} & & \\
\hline Edad (años) & $\mathbf{N}$ & Media & DE & F & Sig. \\
\hline $0-3$ & 5 & 0.45 & 0.078 & & \\
$4-7$ & 22 & 0.393 & 0.244 & & \\
\hline $8-11$ & 38 & 0.449 & 0.23 & 1.127 & 0.349 \\
$12-15$ & 24 & 0.413 & 0.26 & & \\
$16-18$ & 11 & 0.287 & 0.144 & & \\
Total & 100 & 0.41 & 0.23 & & \\
\hline
\end{tabular}


Al estratificar la muestra de acuerdo con el género para determinar el tipo de flujo salival predominante, no se mostró una diferencia estadísticamente significativa entre el género masculino y femenino (Cuadro 7).

El tipo de flujo que prevaleció en todos los grupos de edad fue nuevamente el pronunciadamente bajo. En consecuencia, al aplicar la prueba $X^{2}$ no se encontró diferencia estadísticamente significativa alguna, de acuerdo con la edad (Cuadro 8).

\section{Relación entre el pH salival y la TFS}

Con el propósito de determinar si este estudio mostró una correlación entre el $\mathrm{pH}$ salival y la TFS, se aplicó el coeficiente de correlación de Pearson. Esta prueba indicó que no existió una correlación estadísticamente significativa entre el pH salival y la TFS ( $r=.163 ; p=.104)$, como lo muestra la Figura 1.

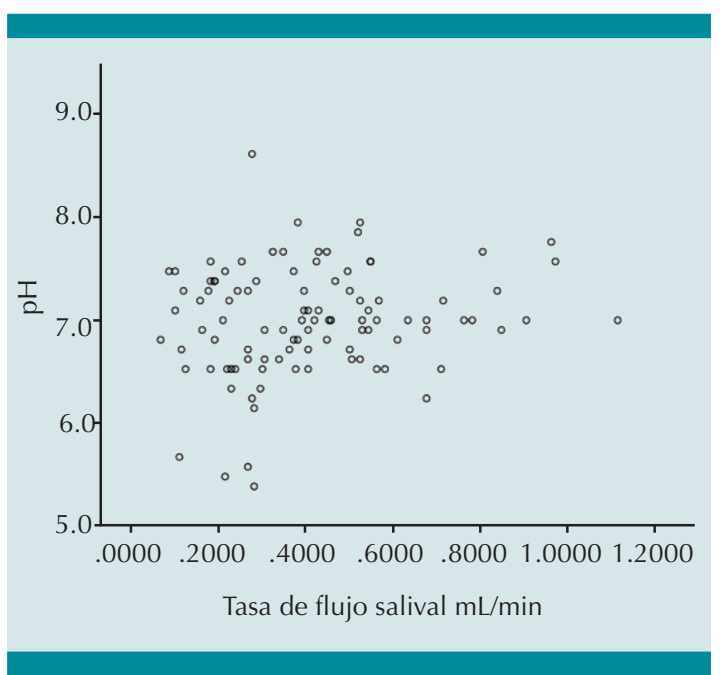

Figura 1. Gráfica de dispersión entre el pH y la TFS.

\section{DISCUSIÓN}

Ha sido documentado el rol crítico que tiene la saliva en la prevención de la caries dental, puesto que se encarga de regular el equilibrio

Cuadro 7. Frecuencias de los tipos de flujo salival de acuerdo con el género

\begin{tabular}{|c|c|c|c|c|c|c|c|c|}
\hline \multirow[b]{2}{*}{ Género } & \multirow[b]{2}{*}{$\mathbf{n}$} & \multicolumn{5}{|c|}{ Tipo de flujo salival } & \multirow[b]{2}{*}{$\mathrm{X}^{2}$} & \multirow[b]{2}{*}{ Sig. } \\
\hline & & Xerostomía & Pronunciadamente bajo & Bajo & Normal & Hipersalivación & & \\
\hline Masculino & 50 & 1 & 40 & 7 & 2 & 0 & & \\
\hline Femenino & 50 & 4 & 44 & 2 & 0 & 0 & 6.768 & 0.08 \\
\hline Total & 100 & 5 & 84 & 9 & 2 & 0 & & \\
\hline
\end{tabular}

Cuadro 8. Frecuencias de los tipos de flujo salival de acuerdo con la edad

\begin{tabular}{|c|c|c|c|c|c|c|c|c|}
\hline \multirow[b]{2}{*}{ Edad (años) } & \multicolumn{6}{|c|}{ Tipo de flujo salival } & \multirow[b]{2}{*}{$\mathrm{X}^{2}$} & \multirow[b]{2}{*}{ Sig. } \\
\hline & $\mathbf{n}$ & Xerostomía & Pronunciadamente bajo & Bajo & Normal & Hipersalivación & & \\
\hline $0-3$ & 5 & 0 & 5 & 0 & 0 & 0 & \multirow{6}{*}{5.356} & \multirow{6}{*}{0.945} \\
\hline $4-7$ & 22 & 1 & 18 & 3 & 0 & 0 & & \\
\hline $8-11$ & 38 & 1 & 32 & 4 & 1 & 0 & & \\
\hline $12-15$ & 24 & 2 & 19 & 2 & 1 & 0 & & \\
\hline $16-18$ & 11 & 1 & 10 & 0 & 0 & 0 & & \\
\hline Total & 100 & 5 & 84 & 9 & 2 & 0 & & \\
\hline
\end{tabular}


entre la desmineralización y la remineralización en un ambiente cariogénico, adicionalmente la TFS y la viscocidad de la saliva pueden influir en el desarrollo de caries dental, ya que la saliva tiene propiedades antibacteriales y anti infecciosas y se ha demostrado que una TFS menor a $0.7 \mathrm{~mL} / \mathrm{min}$ puede incrementar el riesgo de la destrucción del órgano dentario; ${ }^{10}$ sin embargo, en los niños con SD, la saliva puede presentar alteraciones físicas y químicas que reducen su función protectora sobre la superficie de los dientes. Respecto a esto, se han reportado niveles altos de capacidad amortiguadora y $\mathrm{pH}$ salival y valores bajos de TFS en estos niños. ${ }^{11}$

En los estudios realizados sobre saliva en niños con SD, se ha utilizado tanto saliva no estimulada como saliva estimulada. En el presente estudio se decidió utilizar saliva no estimulada, tomando en cuenta que ésta puede aportar información valiosa y relacionarse directamente con las condiciones clínicas del paciente, de forma más precisa que la saliva estimulada. ${ }^{12}$

El-Yazeed, Taha, El Shehaby y Salem en el 2009 reportaron alteraciones en la composición salival y una reducción del $\mathrm{pH}$ salival en niños con SD. Siqueira, Bermejo, Mustachi y Nicolau en el 2005 y Raurale, Viddyasagar, Dahapute, Joshi y Badakar en el 2013, en estudios realizados en niños con SD, demostraron que no existieron diferencias significativas en los valores promedio de $\mathrm{pH}$ salival entre niños y niñas, por lo que los resultados obtenidos en la presente investigación coinciden con los resultados de estos autores; no obstante, se encontraron valores más altos en el género femenino. $\mathrm{El} \mathrm{pH}$ de tipo ácido fue el más frecuente y se observó que los valores de pH eran más altos conforme la edad aumentaba, esto último se observó a pesar de que el grupo de 16-18 años de edad fue el más reducido en cuanto al número de niños con SD. ${ }^{12-14}$

En lo que se refiere a la TFS, este valor tendió a disminuir ligeramente conforme la edad aumen- taba, aunque estadísticamente esta diferencia no fue significativa. El TFS más prevalente, fue el pronunciadamente bajo, y se reportaron valores más altos en el género masculino, por lo que estos resultados coinciden con los resultados obtenidos por Freire, Pardi y Pereira en su estudio realizado en saliva en el 2011 en niños con SD. ${ }^{9}$

En cuanto a la relación entre el pH y la TFS, se ha demostrado en estudios previos que esta relación no es directamente proporcional, ya que el hecho de que se observe un valor alto en el $\mathrm{pH}$ no necesariamente significa que habrá una TFS elevada, o por el contrario, el que se encuentre un valor bajo de TFS, no siempre significa que habrá un pH salival bajo; tal es el caso de los resultados obtenidos en este estudio en el que a pesar en encontrarse que los valores de $\mathrm{pH}$ aumentaban conforme la edad y de que la TFS por el contrario disminuía, no se encontró una correlación estadísticamente significativa.

Por otro lado, es importante reconocer que entre las limitaciones del presente estudio se encuentra que no se realizó una selección aleatoria de la muestra, ya que no fue posible incluir todas las escuelas de educación especial ubicados en la ciudad de Mérida, Yucatán, México, por lo tanto, los resultados obtenidos no pueden generalizarse a todos los niños con SD inscritos en estas escuelas y el numero de niños incluidos por grupo de edad no fue el mismo en todos los grupos, lo cual pudiera representar un sesgo en los resultados obtenidos cuando se compararon las variables entre los grupos de edad.

Otra limitación sería que no se estudiaron variables que pudieran influir en los niveles de $\mathrm{pH}$ y TFS, además del sexo y la edad. Por lo que, para futuras investigaciones se sugiere realizar una selección aleatoria de los participantes, incrementar el tamaño de la muestra y homogenizarla en edad y sexo, así como considerar un grupo control y correlacionar estas características 
salivales con patologías como la caries dental, para determinar si los valores encontrados en ellas influyen en los niveles de caries dental que presentan estos niños, con base en lo reportado en la literatura.

\section{CONCLUSIÓN}

En este estudio no se encontraron diferencias estadísticamente significativas en el valor de $\mathrm{pH}$ salival entre niños y niñas y éste aumentó a mayor edad; sin embargo, predominó el pH salival ácido; por el contrario, sí se encontraron diferencias significativas en la TFS entre niños y niñas con SD y predominó la TFS pronunciadamente baja y a pesar de que no se encontró una correlación estadísticamente significativa entre el pH salival y la TFS, se puede concluir que los valores de $\mathrm{pH}$ salival y TFS deben considerarse como indicadores de salud durante la consulta odontológica del paciente con SD para establecer el diagnóstico de su estado de salud bucal, de tal manera que se proporcione el tratamiento más adecuado en cada caso.

\section{AGRADECIMIENTOS}

A todas las instituciones de atención al paciente con SD en las cuales se realizó esta investigación y a los padres que otorgaron su consentimiento para la participación en este estudio de sus hijos.

\section{REFERENCIAS}

1. Instituto Nacional de Estadística, Geografía e Informática (México). Clasificación de tipo de discapacidad [internet]. [Ciudad de México]: Dirección General de Estadística (México); Instituto Nacional de Estadística, Geografía e Informática; 2000 [Revisada 2010].[28 p.]. Disponible en: http://www.inegi.org.mx/est/contenidos/proyectos/ aspectosmetodologicos/clasificadoresycatalogos/doc/ clasificacion_de_tipo_de_discapacidad.pdf
2. Normastura A. Saliva and dental caries in Down syndrome Children. Sains Malaysiana. 2013;42(1):59-63.

3. Franco G, Saab R, Pizzato LV, Torres MF, Fregoneze AP, Brancher JA. Analysis of salivary $\mathrm{pH}$, flow rate, buffering capacity, concentrations of calcium, urea and total proteins in 2-8 years-old children with Down's syndrome. RSBO. 2014;11(1):66-70.

4. Nederkoorn C, Smulders FT, Jansen A. Recording of swallowing events using electromyography as a non-invasive. Measurement of salivation. Appetite, 1999;33:361-9.

5. Davidovich E, Aframian DJ, Shapira J, Peretz B. A comparison of the sialochemistry, oral $\mathrm{pH}$, and oral health status of Down syndrome children to healthy children. International Journal of Paediatric Dentistry. 2010;20:235-41.

6. Chaushu S, Nof EY, Becker A, Shapira J, Chaushu G. Parotid salivary immunoglobulins, recurrent respiratory tract infections and gingival health in institutionalized and noninstitutionalized subjects with Down's syndrome. Arch Oral Biol. 2002;101-8.

7. Areias CM, Sampaio-Maía B, Macho V, Leal I, Melo P, De Andrade $C$. Does the chemistry in the saliva of Down syndrome children explain their low caries prevalence? European Journal of Paediatric Dentistry. 2013;14(1):23-6.

8. Almerich J M, López P, Silvestre J, Rioboo R, Baca P. Simposio sobre: Saliva y salud dental. Sociedad Española de Epidemiología y Salud Pública Oral (SESPO). España: PROMOLIBRO; 1998:41.

9. Freire AR, Pardi V, Pereira CV. Dental caries experience in relation to salivary findings and molecular identification of S. mutans and S. sobrinus in subjects with Down syndrome. Odontology. 2011;99:162-7.

10. Siqueira L, Moustacchi Z., Nicolau J. Electrolyte concentrations in saliva of children aged 6-10 years with downs syndrome. Oral Surg oral med oral pathooral radiol. 2004;98(2):76-79.

11. Soham B, Srilatha KT, Seema D. Salivary characteristics of Down's síndrome children- A review. J Oral Hyg Heatlh. 2016;4(2):1-3.

12. El-Yazeed A, Taha S, El Shehaby F, Salem G. Relationship between salivary composition and dental caries among a group of Egyptian Down syndrome Children. Aust J Basic \& Appl Sci. 2009;3(2):720-30.

13. Siqueira WL, Bermejo PR, Mustacchi Z, Nicolau J. Buffer capacity, $\mathrm{pH}$, and flow rate in saliva of children aged 2-60 months with Down syndrome. Clin Oral Invest. 2005;9(1):26-9.

14. Raurale A, Viddyasagar M, Dahapute S, Joshi S, Badakar C. Evaluation of oral health status, salivary characteristics and dental caries experience in Down's syndrome children. NJIRM. 2013;4(6):59-65. 